\title{
THE RELATIONSHIP BETWEEN MAJOR GENE CONTROLLED INFLORESCENCE MORPHOLOGY AND CONTINUOUS VARIATION FOR FINAL HEIGHT IN NICOTIANA RUSTICA
}

\author{
J. L. JINKS and D. T. COOMBS \\ Department of Genetics, University of Birmingham, Birmingham BI5 2TT, U.K.
}

Received 2.xi.78

\begin{abstract}
SUMMARY
Inflorescence morphology is a major discontinuity among Nicotiana rustica varieties and is controlled by major genes at, at least, two loci, which display duplicate non-allelic interactions. Its contribution to continuous variation for final height has been examined in $F_{1}, F_{2}$ and backcross families of a diallel set of crosses among eight inbred lines and in the $F_{8}$ families of one of the crosses. It makes no direct contribution, but if all plants are divided into mop and nonmop types of inflorescence most of the variation in final height within each type can be accounted for by the variation in flowering time. Inflorescence morphology appears to switch the correlation between flowering time and final height from a very high negative value within the mops to a very high positive value within non-mops. Most of the non-allelic interactions for final height occur in crosses between a mop and a non-mop parent.

These results imply that the major genes controlling inflorescence morphology exercise a critical control over the continuous variation for final height. However, to explain the lower correlations in the $F_{8}$ families and the positive correlations among mop head varieties not included in the diallel it is necessary to assume that allelic differences at loci that are not concerned with inflorescence morphology are also involved in the switch.
\end{abstract}

\section{INTRODUCTION}

RECENTLY there has been renewed interest in the nature of the determinants of continuous variation. Contributory causes have been new and direct evidence (Jinks and Towey, 1976; Towey and Jinks, 1977) that in continuous variation the effective factor, typically a linked complex of genes, is the unit of segregation (Mather, 1949; Mather and Jinks, 1971, 1977) and the strong criticism that has recently been levelled at this basic and long-standing concept (Kempthorne, 1977; Hill and Avery, 1978). It has also arisen in part from the recent identification of genes or effective factors that make relatively major contributions to the variation, or major genes that make a pleiotropic contribution of relatively minor effect (Oliverio, 1974; Gale and Marshall, 1975; Caten, 1977; Gale, 1977).

The difference between the dense inflorescence of a mop head and the elongate inflorescence of a non-mop head is the only major gene controlled discontinuity among the world-wide collection of over 200 inbred varieties of Nicotiana rustica maintained at Birmingham that affects any of the many characters which have been shown to display continuous variation. In this paper we shall examine the influence of inflorescence morphology on final height, which is the largest component of the variation in $\mathcal{N}$. rustica, in crosses between mop and non-mop headed varieties. At the same time we shall present new data on the inheritance of inflorescence morphology itself.

$$
42 / 3-B
$$




\section{Material}

Three sets of material will be referred to in this paper. The first is a diallel set of crosses consisting of eight pure-breeding lines (three, referred to as lines 1, 3 and 6 are mop head and five, referred to as lines 2, 4, 5, 7 and 8 are non-mop head) and all possible $F_{1}$ crosses between them including reciprocals raised in 1951, 1952, and 1953. These were supplemented in 1952 and 1953 by all possible $F_{2}$ and first backcross $\left(B_{1}\right.$ and $\left.B_{2}\right)$ families derivable from these $F_{1}$ 's and parents. Details of the experimental design have been given by Jinks $(1954,1956)$. Of the characters scored those of interest are final height, flowering time and mop-headedness.

The second is the $\mathbf{F}_{2}$ and $F_{9}$ of the cross between varieties 2 and 12 (lines 1 and 4 of the diallel experiment) raised in 1977 (Boughey, Jinks, Coombs and Shufflebotham, 1978). The data of interest are flowering time and the segregation of mop-headedness in a random sample of $900 \mathrm{~F}_{2}$ plants and $60 \mathrm{~F}_{9}$ families each of 15 plants (Boughey, 1978) and their final heights and growth curves reported by Boughey, Jinks, Coombs and Shufflebotham (1978).

The third is eight plants of each of 75 pure-breeding varieties of $\mathcal{N}$. rustica raised in a completely randomised experiment in 1977 and scored for flowering time, final height and mop-headedness. Further details of these varieties are given in section 4.

\section{Results}

\section{(i) Summary analyses}

The salient features of the extensive analyses of final height in the $8 \times 8$ diallel set of crosses are summarised in fig. 1 as $\mathrm{Wr}$ (array covariance) and Vr (array variance) graphs standardised by expressing all values as percentages of $\mathrm{Vp}$ (variance of the parental family means). For reasons that

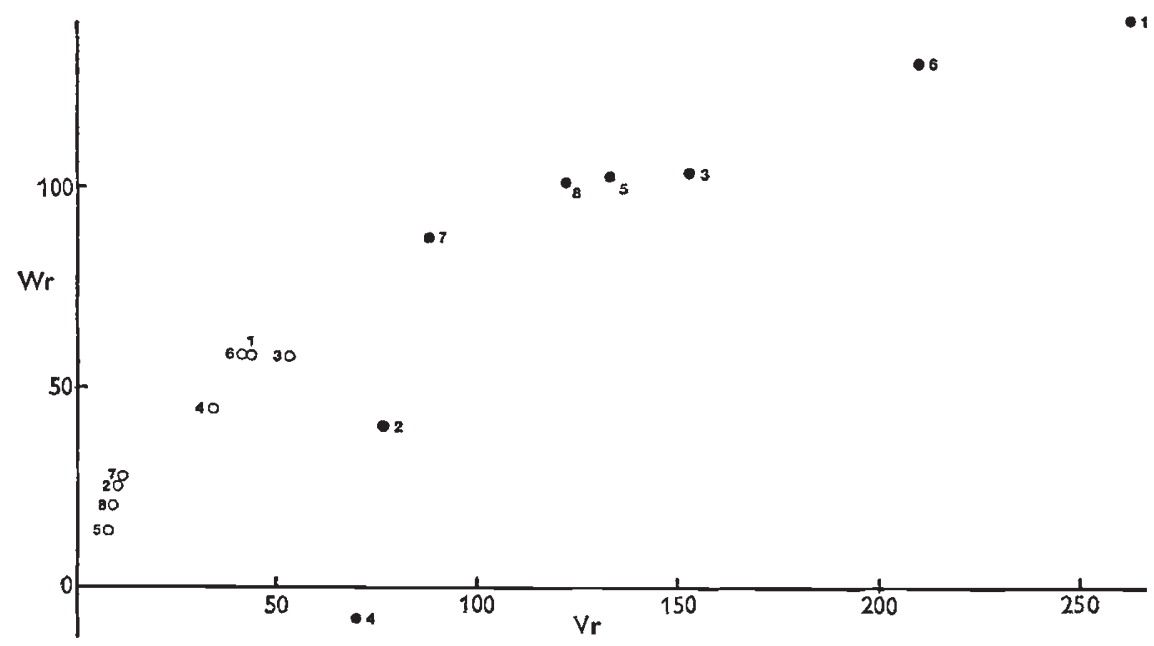

Fig. 1.-Wr, Vr graphs standardised on the variances of the parents $(\mathrm{Vp}=100)$ for flowering time (O) and final height (O). The eight array points for flowering time do not deviate significantly from a linear slope of $0.932 \pm 0.093$ or from the expected slope of one. For final height they deviate significantly from the best fitting linear slope of $0.597 \pm 0.165$ which is also significantly less than the expected slope of one. 
will emerge later the corresponding graph for flowering time is also included. The estimates of $\mathrm{Wr}, \mathrm{Vr}$ and $\mathrm{Vp}$ are based upon parental and $\mathrm{F}_{\mathbf{1}}$ family means averaged over replicate plants, blocks, reciprocals and the 1951, 1952 and 1953 seasons. The two graphs present a now familiar picture of the genetical control of final height and flowering time that is consistent over replications (Jinks, 1954, 1956).

Since the spread of the arrays (1-8) on the graph is due entirely to nonadditive gene action (dominance and non-allelic interactions) the standardisation on $\mathrm{Vp}$, which is the sum of the additive genetic (D) and additive environmental (E) components, brings out the relatively much greater nonadditive component for final height compared with flowering time. For final height the $\mathrm{Wr}$ and $\mathrm{Vr}$ estimates in excess of the value of $\mathrm{Vp}$ (standardised to equal 100 in fig. 1) and the trend towards negative value of $\mathrm{Wr}$ as $\mathrm{Vr}$ tends to zero indicate either overdominance (dominance component $\mathrm{H}>\mathrm{D}$ ), non-allelic interactions, or both. On the other hand, for flowering time the absence of $\mathrm{Wr}$ and $\mathrm{Vr}$ estimates approaching $\mathrm{Vp}$ in value and the positive values of $\mathrm{Wr}$ as $\mathrm{Vr}$ approaches zero indicate partial dominance $(\mathrm{D}>\mathrm{H}>0)$ only.

There is, however, internal evidence from the graphs, strongly supported by independent evidence from joint scaling tests (Jinks, 1956; Jinks and Jones, 1958), that dominance is not the only source of non-additivity responsible for the distribution of the array points on the $\mathrm{Wr}, \mathrm{Vr}$ graphs. Again the evidence is much stronger for final height where the array points scatter widely and significantly from the linear regression line of unit slope which is expected if dominance were the sole source of non-additivity. Additional evidence bearing on the distribution and nature of the non-allelic interaction for final height is presented in section 3 (iii).

For flowering time the array points on the $\mathrm{Wr}, \mathrm{Vr}$ graph do not deviate significantly from a linear regression of unit slope. Nevertheless they clearly follow a curve, rather than a straight line, of the kind that can arise from a linkage disequilibrium in which there is an excess of genotypes with like alleles associated or from non-allelic interactions of a duplicate kind (Jinks, 1956; Jinks and Jones, 1958; Hill, 1964; Mather, 1967). Independent evidence from the joint scaling tests suggests a low level of non-allelic interactions that is variable in its occurrence between seasons.

With the high level of non-allelic interactions for final height it would be hazardous to draw conclusions about the direction of dominance or the distribution of dominant alleles among the eight parental lines. For flowering time, however, the later flowering mop headed varieties 1,3 and 6 also have the least dominant alleles and the earlier flowering varieties 2, 5, 7 and 8 the most, with variety 4 intermediate in both respects.

Overall there is no correlation between final height and flowering time in these data (table 1). Nevertheless before leaving the $\mathrm{Wr}, \mathrm{Vr}$ graphs it is worth noting that the three mop headed varieties 1,3 and 6 have larger $\mathrm{Wr}, \mathrm{Vr}$ values and are, therefore, more recessive than the non-mop heads for both characters.

Although major gene differences are involved in the difference between mop and non-mop heads, the genetical control is not simple. The most critical examination has been made in the $\mathrm{F}_{2}$ and $\mathrm{F}_{9}$ generations of the cross between varieties 2 and 12 ( 1 and 4 of the $8 \times 8$ diallel). In 1977 Hilarie Boughey raised $900 \mathrm{~F}_{2}$ plants and 15 plants of each of $60 \mathrm{~F}_{9}$ families (900 
TABLE 1

The correlation between flowering time and final height in the diallel set of crosses between eight purebreeding varieties of Nicotiana rustica

$\begin{array}{lccr}\text { Material } & \text { Overall } & \text { Mops } & \text { Non-mops } \\ \text { Parental varieties } & 0.364 \text { n.s. } & -0.999 * * & 0.963 * * * \\ \text { Diallel set } & 0.099 \text { n.s. } & -0.806 * & 0.576 * * * \\ \quad \text { n.s.: } \mathbf{P}>0 \cdot 10 ; * \mathbf{P}=0.05-0 \cdot 10 ; * * \mathbf{P}=0.01-0.05 ; * * * \mathbf{P}<0.01 .\end{array}$

plants in all) in a completely randomised experiment and scored them for mop-headedness using similarity to contemporary mop headed varieties as the criterion. She obtained an 846:54 segregation of non-mop to mop head in the $F_{2}$ and a $52: 8$ ratio of non-mop to mop headed families among the $60 \mathrm{~F}_{9}$ families. The $F_{2}$ segregation is the $15: \mathrm{I}$ expected for two loci displaying duplicate gene interaction with mop head as the double recessive $(P=0 \cdot 80)$. The $F_{9}$ ratio, however, is closer to the $7: 1$ expected from three loci displaying triplicate gene interaction $(P>0.90)$ than to the $3: 1$ expected from duplicate genes $(\mathbf{P}=0 \cdot 05-0 \cdot 02)$. This probably reflects the loss of distinctiveness between mop and non-mop headed types that has characterised all previous studies of its segregation in the more advanced generation of a cross and which can only be attributed to the influence of modifier genes at other loci. One thing is clear, however, to be unambiguously classifiable as a mop head a segregant of this cross must be homozygous recessive at, at least, two and possibly at three or more loci.

\section{(ii) Relationship between mop head, flowering time and final height}

We have already noted (table 1) that flowering time and final height are not correlated within the $8 \times 8$ diallel. There is also no significant correlation between these two characters within the eight varieties used as parents. Furthermore, there is no significant difference in final height between mop and non-mop heads either among the eight varieties or among the $8 \times 8$ diallel set $(P>0.05)$. Thus no significant part of the continuous variation for final height can be attributed to the pleiotropic action of the major genes controlling inflorescence morphology or of the genes controlling flowering time. If, however, the eight varieties are divided into mop and non-mop heads there are very highly significant correlations between flowering time and final height within each, but they are of opposing sign (table 1). Among the mop heads the relationship is negative, early flowering leading to taller plants while among the non-mop heads the relationship is positive, early flowering leading to shorter plants. If we allow for this interaction between mop-headedness and flowering time these two characters jointly account for 98.1 per cent of the variation in final height among the eight inbred varieties.

A similar situation exists within the full $8 \times 8$ diallel set of crosses. If we again divide the families into mop head and non-mop head there is a high negative correlation of borderline significance between flowering time and final height within the former and a highly significant positive correlation between these characters within the latter (table l) and if we take this interaction into account, type of inflorescence and flowering time jointly account for 93.8 per cent of the variation for final height.

Exactly half of the families with a non-mop head inflorescence are the 
progeny of non-mop headed parents, and half are the progeny of a cross between a mop headed and a non-mop headed parent. We can, therefore, examine whether homozygosity as opposed to heterozygosity at the loci controlling type of inflorescence also affects the relationship between final height and flowering time. The correlation between these characters is positive within each group but it is significantly greater among the heterozygotes $(P=0.02)$.

\section{(iii) Non-allelic interaction for final height}

It is clear from our analyses that while inflorescence morphology has no direct influence on final height it acts as a major switch on the developmental relationship between flowering time and final height. As a result this relationship differs according to whether a family has two mop headed parents, two non-mop headed parents or one mop and one non-mop headed parent. It is appropriate, therefore, to re-examine the variation for final height in these three kinds of families by subdividing the $8 \times 8$ diallel set into the $3 \times 3$ set of crosses between mop headed parents, the $5 \times 5$ set of crosses between non-mop headed parents and the remaining $5 \times 3$ set of crosses between a mop and a non-mop headed parent.

Re-analysis of the $3 \times 3$ and $5 \times 5$ sets of crosses yields $\mathrm{Wr}, \mathrm{Vr}$ values that are linearly distributed with linear regression slopes that do not differ significantly from one $(P=0 \cdot 20-0 \cdot 10$ and $>0 \cdot 90)$. Furthermore, a joint regression line of slope $0.8225 \pm 0.0818$, which is not significantly different from one, satisfactorily fits both sets of $\mathrm{Wr}, \mathrm{Vr}$ points. There is, therefore, no evidence that a model which assumes that all the non-additive gene action is attributable to dominance is inadequate. From the position of the best fitting line it is clear that dominance is no more than complete, overdominance is ruled out, and from the distribution of array points on the line dominance is in the direction of greater final height.

The results of re-analysing the $3 \times 5$ set of crosses between mop and non-mop headed varieties are in complete contrast. The three pairs of $\mathrm{Wr}$, $\mathrm{Vr}$ values for the mop-headed varieties and the five pairs of values for the non-mop headed varieties have as markedly a non-linear distribution as those from the full $8 \times 8$ diallel. The regression slopes are both significantly less than one $(\mathrm{P}=0 \cdot 01-0 \cdot 001$ and $0 \cdot 05-0.02)$ while a joint regression slope of $0.3779 \pm 0.0226$, which is also significantly less than one, fits both sets of $\mathrm{Wr}, \mathrm{Vr}$ values. Clearly a model that assumes that all the non-additive effects are due to dominance only, fails more conclusively in these crosses than it did in the full diallel.

Independent evidence of the incidence and magnitude of non-allelic interactions for final height is provided by the combined analyses of the parental, $F_{1}, F_{2}$ and first backcrosses $\left(B_{1}\right.$ and $\left.B_{2}\right)$ of each cross in the $8 \times 8$ diallel in the 1952 and 1953 seasons (section 2). This evidence has been presented in summary in two publications (Jinks, 1956; Jinks and Jones, 1958). If we first consider only the ten crosses which display significant non-allelic interactions on the joint scaling tests consistently in both 1952 and 1953 we find that eight are crosses between the three mop headed varieties (1, 3 and 6) and three of the five non-mop headed varieties $(2,4$ and 8 ); none is a cross between a pair of mop heads. Similarly if we consider the additional nine crosses which give some evidence of non-allelic interactions on at least one scaling test in at least one season we find that 
four are crosses between a mop and a non-mop headed variety. Again none is a cross between two mop heads. Thus the independent evidence also suggests that non-allelic interactions, and in particular those that are sufficiently large and consistent to be readily detected in both seasons, occur mainly in crosses between mop and non-mop headed varieties.

\section{(iv) The V2 $\times$ V12 cross}

The cross between varieties 2 and 12 (lines 1 and 4 of the diallel) is typical of those between a mop and a non-mop headed parent that display high and consistent non-allelic interactions for final height. As already noted (section 3 (i)) the 900 plants in this $F_{2}$ segregate in the proportions 15 non-mop : 1 mop as expected if controlled by genes at two unlinked loci displaying non-allelic interactions of a duplicate kind. Over the whole $\mathbf{F}_{2}$ there is as usual no correlation between flowering time and final height $(r=0.099)$. Among the mops, however, there is the characteristic highly significant, negative correlation. The magnitude of this correlation $(-0.3713)$ is less than observed among the mop varieties (table 1) but this is only to be expected as the latter was based on family means replicated 12 times while the former is of necessity based upon observations on single plants. This must also have contributed to the non-significance of the correlation for non-mop headed plants although a second factor may be equally responsible, namely, the genetical heterogeneity at the loci controlling type of inflorescence in the $\mathrm{F}_{2}$ plants with the dominant non-mop phenotype. There must be at least eight different genotypes at these loci all of which have the dominant phenotype (section 3 (i)). We have already shown that three genotypes at these loci, the homozygous double recessive, the homozygous double dominant and the double heterozygote, lead to significantly different relationships between flowering time and final height (section 3 (i)). It would be surprising, therefore, if the remaining genotypes that can occur at these loci in an $\mathbf{F}_{2}$ did not affect this relationship. Equally, it would be surprising if any such effect did not, in turn, reduce the overall correlation between flowering time and final height among the non-mop phenotypes in the $F_{2}$. Nevertheless, there is still a significant difference, in the expected direction, between the correlation within the mop and the non-mop phenotypes $(P<0 \cdot 05)$.

Further evidence that genetical heterogeneity among the non-mop phenotypes may reduce the correlation between flowering time and final height comes from the $\mathbf{F}_{\mathbf{9}}$ families of the same cross (section 2). The mop and nonmop headed families display the characteristic negative $(-0.689)$ and positive $(0.242)$ correlations, respectively. Both are lower than in the parents and $F_{1}$ 's of the diallel (table 1 ), that for the non-mops being particularly so. The mops are, of course, genetically homogeneous at the loci controlling type of inflorescence, being the multiple recessive homozygote. The non-mops, on the other hand, are genetically heterogeneous with at least three and perhaps more than seven different homozygotes at the loci controlling type of inflorescence giving this phenotype (section 3 (i)). The relatively low correlation would, therefore, be expected if again some of these genotypes produced different developmental relationships between flowering time and final height. An alternative explanation of the lower correlations in the $\mathrm{F}_{2}$ and $\mathrm{F}_{9}$ families is, however, possible. For example, 
allelic differences at other loci which are not concerned with type of inflorescence could be partly or even wholly responsible for the switch from a high positive to a high negative correlation among the parental varieties and their $F_{1}$ crosses providing that in these families they are associated with the allelic differences controlling inflorescence morphology. They would, however, freely reassort or, if linked, recombine in the $\mathbf{F}_{2}$ and subsequent generations of a cross between a mop and a non-mop headed parent with a resulting loss or reduction in the positive and negative correlations among their non-mop and mop progeny, respectively. Alone, however, this would not account for the greater reduction in the positive correlation among the non-mops which would still require an explanation based on the genetical heterogeneity at the loci controlling type of inflorescence.

Irrespective of the cause of the reduced correlation, however, in the V $2 \times$ V12 cross, type of inflorescence is still significantly associated with the switch in the direction of the relationship between flowering time and final height, while type of inflorescence itself is showing a segregation pattern characteristic of the duplicate (or higher order) type of non-allelic interaction. It seems inevitable, therefore, that in such crosses final height itself will display a pattern of variation that cannot be explained without also involving non-allelic interactions-as is indeed found.

\section{(v) The origin of the correlations}

Examination of the growth curves of the $F_{9}$ families of the cross between varieties 2 and 12 previously reported by Boughey and Jinks (1978) and Boughey, Jinks, Coombs and Shufflebotham (1978) reveals a striking dichotomy between the growth curves of mop and non-mop headed families with a below-average final height. The growth rates of the shorter non-mop heads begin to accelerate by the first weekly height measurement after planting in the field and they complete most of their growth by the fifth measurement. In contrast the growth rate of the shorter mop heads does not begin to accelerate until the fourth weekly height measurement and it is the eighth measurement before most of the growth is completed. In both cases flowering time marks the onset of the deceleration in growth rate so that the shorter non-mops flower early and the shorter mops late. Families with above-average final heights have growth curves that fall between these two extremes. The observed reversal of the correlation of flowering time and final height between mop and non-mop headed genotypes, therefore, follows directly from the shorter non-mop and mop headed genotypes achieving their final heights relatively rapidly and slowly, respectively.

\section{Generality of conclusions}

In 1977, 75 of our current collection of over 200 inbred varieties of $\mathcal{N}$. rustica were raised in a completely randomised experiment (see section 2) and 61 of these were part of the Birmingham collection in 1950 or had been derived from crosses between these varieties. The remaining 14 were new acquisitions of the very tall, late flowering Brasilia type of mop headed variety which we had previously failed to maintain in the collection at Birmingham because of poor seed production. They could not, therefore, be included in the sample of lines used in the diallel. 
The 61 varieties derived from the sampled collection gave correlations between flowering time and final height of 0.028 and 0.650 for mop and non-mop headed varieties, respectively. These differ significantly $(\mathrm{P}<0.05)$ and are in the same direction as the sample although the correlation for the mop varieties is much less extreme. If the 14 Brasilia type mops are now included the correlation within all 26 mop varieties becomes 0.753 and the correlation within the 14 Brasilia type mops alone is 0.728 . Neither of these differs from the correlation among the non-mop varieties. It is clear, therefore, that the highly significant positive correlations found among the non-mop headed families of the $8 \times 8$ diallel applies to all the non-mop headed varieties currently held at Birmingham. In contrast, the highly significant reversal of this correlation found among the mop headed families of the $8 \times 8$ diallel is less marked among the non-Brasilia type of mop head in our collection and does not occur at all among the Brasilia type of mop head.

Type of inflorescence per se cannot therefore be the cause of the reversal of the correlation between flowering time and final height; other genetically controlled characteristics must also be involved which are present in some mop headed varieties but not in others. This conclusion is similar to that reached from considering the correlation in the $F_{2}$ and $F_{9}$ families of the cross between varieties 2 and 12 (section 3 (iv)). Thus whether we increase the range of genotypes over which we examine the correlations by widening the sample of inbred varieties or by allowing new allelic combinations to arise by reassortment and recombination as in the $\mathrm{V} 2 \times \mathrm{V} 12$ cross, the consequences are the same-the discontinuity in inflorescence morphology is no longer the sole determinant of the developmental relationship between flowering time and final height.

In the $8 \times 8$ diallel set of crosses, non-allelic interaction for final height arises with a higher probability and a greater magnitude in crosses between mop and non-mop headed parents presumably because of the epistatic control of the discontinuity in the relationship of flowering time and final height between mop and non-mop headed phenotypes. Of the ten crosses which show a consistently high level of non-allelic interaction for final height, eight are from the 15 crosses between a mop and a non-mop headed parent while only two are from the remaining 13 crosses. Nevertheless, the remaining seven crosses between mop and non-mop headed parents do not show a consistently high level of non-allelic interactions, indeed some give no indication of interaction on any test in any season. It is clear, once again, therefore, that the difference in inflorescence per se cannot be the cause of the non-allelic interactions. Other genetic differences which are associated with it in some parents of the $8 \times 8$ diallel but not others must also be involved. Equally, of course, the occurrence in two crosses between pairs of non-mop headed parents of a consistently high level of non-allelic interactions for final height shows that allelic differences at the loci controlling type of inflorescence is not a prerequisite for the occurrence of these interactions.

In conclusion we have shown that the major discontinuity in inflorescence morphology, while making very little direct contribution to the variation in final height, appears to act as a switch between a high positive and a high negative correlation between flowering time and final height. In the parental and $F_{1}$ families of the $8 \times 8$ diallel almost all of the heritable variation 
in final height which is itself the largest single component of the heritable variation in $\mathcal{N}$. rustica can be accounted for by the variation in flowering time and the data are compatible with inflorescence morphology per se completely controlling this switch. This interpretation, however, has little predictive value beyond these families. Thus on widening the sample of genotypes it is necessary to assume that allelic differences at loci which are not concerned with inflorescence morphology are also involved in order to explain the observed reduction in the correlations. The results illustrate the potential dangers of drawing general inferences about the participation of major genes in the determination of continuous variation on the basis of the early generations of a cross or from a restricted sample of genotypes. It is essentially the failure to recognise the difference between effective factors and genes.

\section{REFERENGES}

BOUGHEY, HILARIE. 1978. Competitive ability and environmental sensitivity in Nicotiana rustica. Ph.D thesis, Univ. of Birmingham.

BOUGHEY, HILARIE, AND JINKS, J. L. 1978. Joint selection for both extremes of mean performance and of sensitivity to a macroenvironmental variable. III. The determinants of sensitivity. Heredity, 40, 363-369.

BOUGHEY, HILARIE, JINKS, J. L., COOMBS, D., AND SHUFFLEBothaM, w. 1978. Joint selection for both extremes of mean performance and of sensitivity to a macroenvironmental variable. IV. Growth pattern and sensitivity. Heredity, 41, 175-183.

CATEN, C. E. 1977. Genetical and physiological analysis of continuous variation in fungi. Heredity, 39, 429.

GALE, M. D. 1977. Genetic analysis and the control of development of height in wheat. Heredity, 39, 429.

GALE, M. D., AND MARShall, G. A. 1975. The nature and genetic control of gibberellin insensitivity in dwarf wheat grain. Heredity, 35, 55-66.

HILL, J. 1964. Effects of correlated gene distributions in the analysis of diallel crosses. Heredity, 19, 27-46.

HILL, w. G., AND AVERY, P. J. 1978. On estimating number of genes by genotype assay. Heredity, 40, 397-404.

JINKS, J. L. 1954. The analysis of continuous variation in a diallel cross of Nicotiana rustica varieties. Genetics, 39, 767-788.

JINKS, J. L. 1956. The $\mathrm{F}_{2}$ and backcross generations from a set of diallel crosses. Heredity, $10,1-30$.

JINKS, J. L., AND JONES, R. M. 1958. Estimation of the components of heterosis. Genetics, 43, 223-234.

JINKS, J. L., AND TOWEY, P. M. 1976. Estimating the number of genes in a polygenic system by genotype assay. Heredity, 37, 69-81.

KEMPTHORNE, O. 1977. In Proceedings of International Conference on Quantitative Genetics, ed. E. Pollak, O. Kempthorne and T. B. Bailey, Jr. Iowa State Univ. Press, Ames.

MATHER, K. 1949. Biometrical Genetics. 1st edn. Methuen, London.

MATHER, K. 1967. Complimentary and duplicate gene interactions in biometrical genetics. Heredity, 22, 97-103.

Mather, K., AND JiNks, J. x. 1971. Biometrical Genetics. 2nd edn. Chapman and Hall, London.

MATHER, K., AND JINKS, J. x. 1977. Introduction to Biometrical Genetics. Chapman and Hall, London.

OLIVERIO, A. 1974. Genetic factors in the control of drug effects on the behaviour of mice, in Genetics of Behaviour, ed. J. H. F. van Abeelen, pp. 375-395. North Holland Publishing Co., Amsterdam.

TOWEY, P. M., AND JINKS, J. L. 1977. Alternative ways of estimating the number of genes in a polygenic system by genotype assay. Heredity, 39, 399-410. 